\title{
La evaluación de las narraciones escritas: una perspectiva holística focalizada
}

Ricardo Benítez Figari'

rbenitez@ucv.cl-

\section{RESUMEN}

Este trabajo tiene por objetivo presentar uno de los cinco instrumentos que se utilizarán en la investigación FONDECYT n. ${ }^{\circ}$ 1070333, con el cual se evaluará la producción escrita de 400 niños y niñas sin trastornos del lenguaje. Dicho instrumento es una pauta de evaluación holística focalizada que se diferencia de la holística pura o simple, con la cual se intenta ya sea clasificar una composición escrita para hacerla corresponder con una serie graduada de otras composiciones 0 asignar puntaje al dominio de ciertos rasgos relevantes al tipo de texto escrito con una pauta que describe cada rasgo e identifica niveles de calidad alta, media y baja. El instrumento que se presenta aquí es una pauta de evaluación que abarca características tanto generales de una producción escrita como específicas de la tipología textual solicitada (narración).

\section{Palabras clave}

Evaluación holística focalizada, rúbrica, puntaje de rasgo primario, respuesta.

\section{ABSTRACT}

The aim of this work is to present one of five instruments that will be employed in the research mentioned above. The written production of 400 children with no learning disabilities will be assessed through a holistic focalized guideline, which is different from the pure or simple holistic assessment, whose objective is to classify a written composition to make it match with a graded series of other compositions or to assign a score to the domain of certain features relevant to the type of written text with a guideline that describes each feature and identifies high, medium and low quality levels. The guideline that will be employed is also different from the Primary Trait Score, which directs the test-giver's attention to those characteristics relevant to the type of discourse required and in which not carrying out the instructions to do the task is severely penalized. Focused Holistic Assessment, instead, is a fusion of both, Holistic Assessment and Primary Trait Scoring, and is an assessment instrument that satisfactorily harmonizes with the production of narrative texts. It assesses the general quality of written texts, regardless of how the producers of those texts respond to specific aspects of a given topic. The instrument presented herein is an assessment guideline that addresses both general characteristics of writing and specific characteristics of the text type required (narration).

\section{KEYWORDS}

Focused Holistic Assessment, rubric, Primary Trait Scoring, response.

\footnotetext{
1 Instituto de Literatura y Ciencias del L̇enguaje, Pontificia Universidad Católica de Valparaíso, Chile. El presente trabajo se enmarca dentro del Proyecto fONDECYT n. ${ }^{\circ} 1070333$.
} 


\section{Introducción}

$\mathrm{E}$ 1 presente trabajo sugiere una evaluación holística focalizada (White y Venneman, 2000) aplicada a narraciones escritas por niños entre 8 años y 8 años y 11 meses que cursan el tercer año de enseñanza básica. El trabajo se enmarca dentro de un proyecto de mayor envergadura del Fondo para el Desarrollo de.la Ciencia y la Tecnología, con el cual se intenta: (a) descubrir si las habilidades metalingüísticas y/o cognitivas se relacionan con el desarrollo de la comprensión oral del lenguaje figurativo en la edad escolar y (b) determinar si existen diferencias sistemáticas respecto de la lectoescritura de niños y niñas que exhiben diferentes niveles de comprensión de lo figurativo, es decir, entre buenos y malos comprendedores tanto de lo oral no literal como de lo textual escrito, y entre buenos y malos productores de textos escritos. Dicho proyecto tiene carácter interuniversitario, pues se trabaja en colaboración con la Universidad de La Serena.

En un proyecto anterior (también de FoNDECYT e interuniversitario), se correlacionó el desarrollo de la comprensión de lo oral figurativo -específicamente ironías y actos de habla indirectos- con la producción escrita y la comprensión lectora. Después de evaluar a 900 niños y niñas desde kindergarten a $8^{\circ}$ año básico en dos regiones de Chile, los resultados revelaron una correlación débil entre la producción escrita y la oralidad en los participantes de la investigación. Lo anterior se debió sólo a que sus productos escritos demostraron un escaso dominio de las convenciones de la lengua escrita, las cuales fueron evaluadas en algunas de sus formas microestructurales (nexos hipo y paratácticos y expresiones interoracionales), y se comprobó que están prácticamente ausentes en las composiciones. Se pudo concluir que un niño de trece años es maduro mental y metapragmáticamente, puesto que es capaz de comprender el $80 \%$ de las ironías (según el Instrumento de Medición de Inferencias Pragmáticas diseñado exclusivamente para esa investigación), pero no domina la escritura como un código peculiar y diferente de la oralidad.
En este marco, este trabajo tiene por objetivo presentar uno de los cinco instrumentos que se utilizarán en esta nueva investigación: una pauta de evaluación holística focalizada (EHF), con la cual se evaluará la producción escrita de 400 niños y niñas sin trastornos del lenguaje.

\section{La eHF y otros tipos de evaluación}

La EHF se diferencia de la que se ha denominado holística (pura o simple), en la cual se clasifican composiciones escritas de acuerdo a una serie de otras composiciones, identificando niveles de calidad alta, media y baja. En la evaluación holística el evaluador lee la composición para luego asignarle un puntaje único basado en una impresión general. Para tal efecto, existen rúbricas especialmente diseñadas. Es un método que sirve para indagar más sobre las fortalezas de la producción escrita que sobre sus deficiencias o debilidades, pero no puede proporcionar un diagnóstico porque no refleja componentes que se refieran a rasgos específicos en forma explícita (Ferris y Hedgcock, 1998). La evaluación holística simple exige al evaluador compartir con otros evaluadores los mismos criterios de calidad para clasificar las composiciones y requiere de lectores expertos, por tal razón, es necesario que se capacite a esos otros evaluadores para que puedan establecer los niveles de calidad (buena, media, alta).

Las rúbricas bien diseñadas pueden servir como un marco consistente para proporcionar retroalimentación significativa a los estudiantes con el fin de que éstos revisen sus trabajos. 
La EHF también se diferencia del puntaje de rasgo primario, con el cual el evaluador debe establecer las características del tipo de discurso que se solicita y en el cual se penaliza con severidad el incumplimiento de las instrucciones para llevar a cabo la tarea, sin importar cuán efectivo o cuán estilizado sea lo que se escribió (White y Venneman, 2000). Este tipo de evaluación la realiza sólo un evaluador, quien sigue una pauta previamente diseñada con las características que él busca en las composiciones. Dichas características pueden ser los tres principales aspectos de la situación retórica (tópico, audiencia y propósito del texto) o pueden ser la evaluación de cuán bien el pasado gramatical se usó en un texto narrativo. Para decidir la calificación de una composición, el evaluador sólo fijará su atención en esas características y no en otras que hacen complejo cualquier tipo de texto.

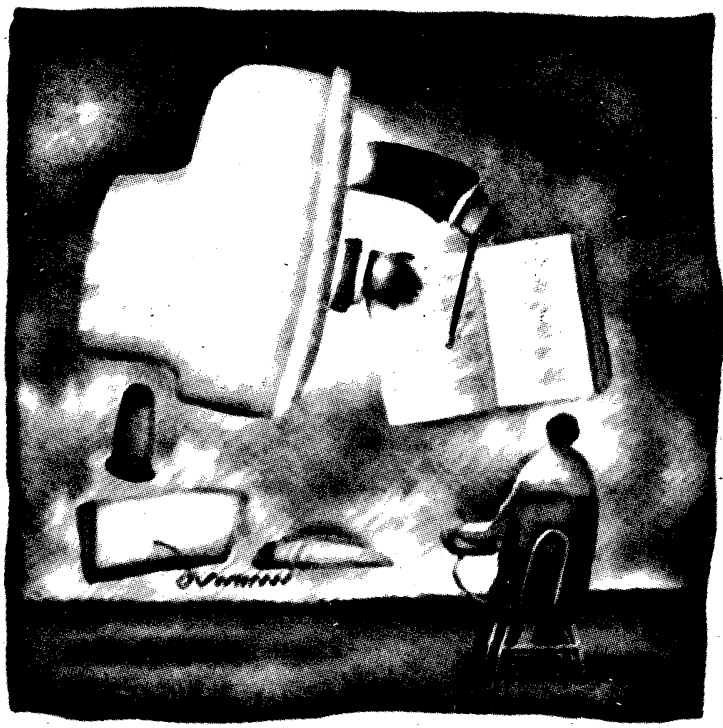

La EHF, en cambio, es una fusión de las dos evaluaciones anteriores, porque combina el trabajo de evaluar las composiciones en forma seriada o graduada entre bajas, medias y altas según su calidad, con rasgos que se consideran importantes en la producción de determinado tipo de texto. Es decir, el evaluador, además de emitir un juicio sobre la calidad de las composiciones, utiliza una rúbrica que le permite clasificarlas de acuerdo a los criterios usados en ella (White y Venneman, 2000).

Por un lado, la EHF permite clasificar los textos escritos de acuerdo a su calidad y, por otro, permite fijar la atención en ciertas características que exige la tarea de escritura. Este tipo de evaluación es un instrumento que armoniza en forma satisfactoria con la producción de textos narrativos, porque permite incorporar los diversos elementos que componen una narración: trama, evolución de los personajes, coherencia de un marco temporal, presencia de un tema y de un contexto, y desarrollo de la comunicación, por ejemplo, consideración de la audiencia, aunque ésta debe ser evaluada en casi todos los otros tipos de texto.

Históricamente, las rúbricas con puntajes se han diseñado como una ayuda para los evaluadores, pero también pueden utilizarse como importantes herramientas para la enseñanza cuando se les dan a conocer a los estudiantes. HampsLyons (1991) sostiene que si los métodos de evaluación dejaran de utilizar sus potencialidades educativas, habría una ruptura entre la enseñanza y la evaluación. Cuando ésta se da a conocer en el proceso de enseñanza, se puede usar una rúbrica como una herramienta efectiva para su proceso, por ejemplo, cuando se presenta a los estudiantes los primeros días de clase, dándoles oportunidades de trabajar con ella antes de que sus criterios se apliquen a sus propios textos. Las rúbricas bien diseñadas pueden servir como un marco consistente para proporcionar retroalimentación significativa a los estudiantes con el fin de que éstos revisen sus trabajos. Para ser usadas productivamente de esta manera, sus descriptores deben ser explícitos, comprensibles y orientados específicamente hacia los niveles de competencia de los estudiantes y hacia los focos retóricos del curso (Ferris y Hedgcock, 1998).

\section{2. ¿Por qué la narración?}

Se sabe que la narración es el tipo de texto que se aprende con mayor facilidad, tal vez por el hecho de haber sido ejercitada oralmente en repetidas ocasiones. Se sabe, además, que la narración es el tipo de texto que demanda menos esfuerzo cognitivo. Sin embargo, sabemos que los niños de tan corta edad (8-9 años) no tienen conciencia de la audiencia a la cual dirigen sus composiciones escritas, principalmente porque 
están en una etapa egocéntrica de su desarrollo. Lo que no se sabe a ciencia cierta és si los niños de este grupo etario pueden mantener el tópico y/o si tienen un propósito claro al narrar:

En una interesante distinción que hace Williams (1998), lo retórico y lo cognitivo aparecen como dos complejidades que, en una secuenciación tradicional, pueden parecer del mismo grado de dificultad. Sin embargo, en un análisis profundo se descubrirá que la narración es tal vez el tipo de texto más exigente retóricamente, aun pudiendo ser menos exigente que la argumentación cognitivamente. La narración tiene un mensaje que demanda un alto nivel de sofisticación retórica y contiene argumentos para una particular representación de la realidad. El objetivo es llevar a los lectores a aceptar la representación vía interacciones de la trama, el personaje, y el contexto. De hecho, una medida del éxito narrativo de un escritor es la credibilidad o aceptabilidad de sus representaciones de la realidad que, de acuerdo con Cooper (1990), debe ser estructurada de la siguiente manera:

El tema de la historia, que puede ser explícito o implícito, es la idea fundamental en torno a la cual gira la totalidad de ella. El argumento, que consta de varios episodios, es la forma en que se organiza la historia. El escenario es el lugary la época en el que sucede la historia. Los personajes son las personas o los animales que participan en la acción de la historia. El problema es la situación en torno a la cual un episodio o la totalidad de la historia se organizan. La acción es lo que sucede como resultado del problema y consta de los hechos conducentes a la solución del problema, a lo que se denomina resolución.

Por otro lado, la producción de textos narrativos es parte fundamental del desarrollo de habilidades para la escritura que se incluyen en el programa de estudios de tercer año de enseñanza básica del Ministerio de Educación de Chile.

De acuerdo con Botting (2002), la habilidad para narrar es una de las maneras más interesantes y ecológicas ${ }^{2}$ en las que se puede medir la

2 Una evaluación ecológica es aquella que "describe, partiendo de los estudios etnográficos, las demandas del entorno y las respuestas de los agentes a ellas, así como los modos múltiples de adaptación. A nivel escolar este competencia comunicativa; puesto que las narraciones forman la base de muchos actos de habla; por su parte, en una visión muy particular, Denning (2005) sostiene que el aprendizaje veloz se produce cuando cerebro y corazón se combinan, y que la narración es fundamental para éste, porque involucra al corazón, desestimando los enfoques abstractos y cerebrales como muy poco eficaces.

La razón de por qué se enseña la narración antes que cualquier otro tipo de texto estriba en la capacidad innata del niño para expresarse. Es por eso que la narración pura -la que, por ejemplo, no apoya un texto argumentativo- se enmarca dentro de la función que se ha denominado expresivismo. Evidentemente, el sentido de.la vista ha ayudado a la expresión con palabras: el niño mira y reconoce antes de que pueda hablar. Es por eso también que una de las primeras expresiones de los niños es el dibujo, que luego verbaliza y finalmente expresa por escrito. Por muchos años, el uso de medios visuales ha ido de la mano con pedagogías expresivistas, tal vez porque los niños y niñas han crecido en lo que a todas luces es una cultura agresivamente visual (George, 2002).

Por su parte, Countryman (1990) revisa las formas y las funciones de las narrativas considerando su influencia en contextos académicos. Estas formas y funciones incluyen:

1. La narrativa terapéutica, como un instrumento para curar viejas heridas y para tratar los males de la vida.

2. La narrativa noética (relacionada o basada en el intelecto o en el pensamiento), como una manera de conocimiento que nos permite traducir la experiencia.

3. La narrativa retórica, como un medio de persuasión, relacionada con los efectos que produce y con la generación de buenas razones.

4. La narrativa pragmática, como un instrumento para lograr metas o fines deseados.

paradigma estudia las situaciones de clase y los modos cómo responden a ellas los individuos, para así tratar de interpretar las relaciones entre el comportamiento y el entorno. La evaluación que defiende este paradigma es sobre todo cualitativa y formativa". Tomado de: http:// www.rmm.cl/index_sub.php?id_seccion=3513\&id_ portal $=5318$ id_contenido $=10145$. 
5. La narrativa estética, como una actividad que llama la atención de nuestros sentidos de la belleza y a la cual atribuimos valor intrínseco.

6. La narrativa ética, como representativa de una posición moral: existen verdades que solo la narrativa puede revelar y esto no es solamente una cuestión de sugestiones como con las alegorías.

7. La narrativa crítica: reforzando la cultura dominante y poniéndola en tela de juicio al mismo tiempo.

Por último, Sharton (1990) señala que los fundamentos para enseñar la narración son, en primer lugar, que la usamos en la vida diaria más allá de la sala de clases, cuando le contamos a algún amigo acerca del día que tuvimos, cuando describimos un evento deportivo, cuando escribimos en diarios personales o en la correspondencia diaria, como el correo electrónico. Narramos problemas que nos afectan cuando escribimos cartas de quejas, anécdotas entretenidas cuando escribimos discursos e historias inspiradoras cuando escribimos sermones. Las narrativas forman la base de los sistemas religiosos, de los mitos y de las leyendas en todo el mundo en documentos como la Sagrada Biblia, el Corán, la Torah, la mitología clásica, como también en la tradición oral de las culturas tribales. Relatos pictóricos se encuentran en los dibujos de las cavernas del Neandertal, en las cámaras de las pirámides, en los frescos de las ruinas romanas, en las galerías de arte y en las campañas publicitarias, como también en las dramatizaciones televisivas y cinematográficas.

\section{Proyecciones}

Se administrará una tarea de escritura a 400 niños y niñas de tercer año básico de escuelas municipalizadas, subvencionadas y particulares de las ciudades de Valparaíso, Viña del Mar y La Serena, Chile. Los participantes tendrán una hora pedagógica (45 minutos) para realizar la tarea, para la cual deberán seguir instrucciones explícitas que darán a conocer ayudantes de la investigación entrenados especialmente para tal efecto. Previo a esto, se piloteará la tarea de escritura con una muestra de aproximadamente
165 estudiantes del mismo nivel de escolaridad. Las instrucciones para realizar la tarea de escritura contienen unas sugerencias para desarrollar tópicos que fueron tomadas de un libro que representó una iniciativa del Ministerio de Educación de Chile, concretizada en un libro cuyo único propósito fue compilar composiciones de niños y niñas chilenos de segundo a sexto año de educación básica (Historias de grandes pequeños), demostrando así cuán creativos y talentosos podían ser. Para efectos de este estudio, las sugerencias fueron tomadas de textos escritos por niños y niñas de tercero básico únicamente, seleccionando aquellos tópicos más recurrentes. Las sugerencias también incluirán un conjunto de imágenes que puedan estimular la producción y que serán tomadas de Internet para proyectarlas en las salas de clases como estímulos visuales. Las instrucciones serán las siguientes:

Seguramente, alguien alguna vez te ha contado un cuento o has leído alguno. En esta ocasión, queremos que tú escribas tu propio cuento, el cual será llevado a padres y niños de otro país que no tienen la posibilidad de escuchar o de leer cuentos como tú. Te damos algunas sugerencias para que inventes tu cuento. Si no te gustan estas sugerencias, puedes crear el tuyo propio con tu imaginación:
Sugerencias:
1. un caballo salvaje
2. niños que podían volar
3. una caja de cuentos
4. hormiguitas
5. superhéroes
6. un programa de $\mathbf{T V}$
7. una película
8. las brujas y los magos
9. los duendes
10. las ciudades perdidas
11. los payasos
12. los extraterrestres
13. lo que pasó en un paseo
14. mis mascotas
15. mis dibujos animados favoritos

Las fotografías que puedes ver en el telón también pueden servirte como fuente de inspiración para tu cuento. 
El hecho de sugerir tópicos a los participantes tiene el único propósito de evitar la pérdida de tiempo en que éstos pueden incurrir al pensar en uno sobre el cual escribir. Los participantes no recibirán ningún tipo de ayuda mientras se realiza la tarea. Se les darán dos hojas de papel, de las cuales una les podrá servir para producir un borrador en caso de que tomen conciencia del proceso de revisión mientras producen el texto pero, una vez más, no se les insinuará que esa hoja extra la pueden usar para el borrador.

La investigación tendrá un carácter netamente descriptivo-cualitativo. $\mathrm{El}$ instrumento será la pauta para la EHF y se capacitará a dos evaluadores más para darle confiabilidad y validez. El instrumento que se presenta a continuación está basado, por una parte, en la producción de un texto narrativo ideal y, por otra, en los trabajos de Cooper y Odell (1977), de Weigle (2000), y de Wolf y Gearhart (1997); estos últimos plantean un continuum para todos los elementos narrativos: tópico, personajes, contexto, trama y comunicación. El tópico puede ir de lo más implícito a lo más explícito, o puede ser más didáctico o más revelador; el personaje central puede ir de lo más plano a lo más pulido, o de lo más estático a lo más dinámico; el contexto puede ser sólo un trasfondo o puede ser esencial, o puede ir de lo más simple a lo más multifuncional; la trama puede ir de lo más simple a lo más complejo, o puede ir de lo más estática a lo más conflictiva; la comunicación puede presentarse desde lo más apegado al contexto hasta demostrar que existe conciencia de una audiencia, y el lenguaje puede ir de lo más literal a lo más simbólico.

A continuación se presenta el instrumento:

\section{Respuesta insatisfactoria}

- El texto no desarrolla una historia (cuento) o es demasiado difícil de entender en conjunto, pues proporciona un mínimo de apoyo o nada de apoyo; puede que sólo haya parafraseado las instrucciones.

- El texto muestra muy poca organización o ninguna.

- El texto no presenta ningún control sobre límites y estructura oracionales; la elección léxica puede ser inexacta, limitada e inmadura en la mayor parte del texto; las oraciones son cortas y simples en estructuras o repetitivas.

- El texto presenta errores ortográficos y de puntuación que impiden severamente la comprensión.

- El autor no es un observador ni un participante en forma sistemática.

- El personaje central no es una persona viva real, sólo es un nombre en la página.

- La acción transcurre sin ningún escenario (contexto) detallado; la acción no se puede ver en un lugar determinado. Contexto con muy poca o ninguna indicación de tiempo o lugar ("Había una niña. Le gustaban los dulces.").

- La escritura está ligada al contexto y a menudo depende del dibujo y de la conversación para clarificar el significado.

- No se entiende qué eventos van primero o después de otros eventos; uno o dos eventos con muy poco o ningún conflicto ("Una vez había un gato. Al gato le gustaba la leche.").

- Uno o dos personajes planos y estáticos, con poca relación entre ellos.

\section{Respuesta insuficiente}

- El cuento es desorganizado, o poco centrado o claro; muy breve.

- Control mínimo sobre límites oracionales y estructuras oracionales; la elección léxica a menudo puede ser inexacta.

- Errores en gramática, ortografía y puntuación interfieren con la comprensión en la mayor parte del texto.

- Producción de sólo el comienzo de un cuento.

- Introduce un personaje nuevo, pero no lo desarrolla más allá de ese punto.

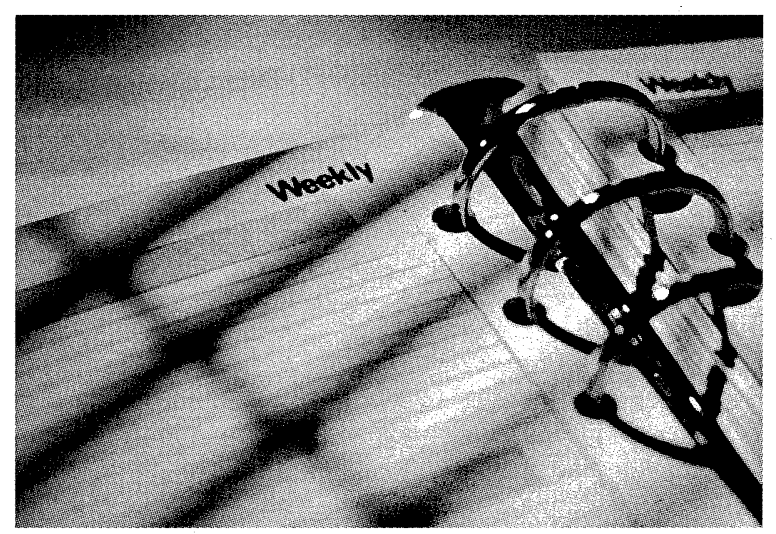


- Alguna clase de pulimento en la descripción física generalmente; la relación entre los personajes está dada por la acción.

\section{Respuesta desequilibrada}

- Puede faltar desarrollo en partes del cuento o ser repetitivo, o sólo es un comienzo bien escrito.

- Es organizada en partes, otras partes están desarticuladas y/o le faltan transiciones.

- Muestra un control disparejo sobre límites oracionales y estructuras oracionales; puede mostrar elecciones léxicas inexactas; palabras comunes y ordinarias se usan de manera tradicional; contiene expresiones trilladas y exageradas.

- Errores en gramática, ortografía y puntuación interfieren a veces con la comprensión.

- Si existe acción dramática, ésta es repetitiva, ya que los eventos no están conectados para formar un cuento coherente.

- A veces el contexto parece vívido y real, pero a veces la acción sólo pasa y el lector no se da cuenta de cuál es el contexto realmente.

- A veces no está claro qué evento sucedió primero.

- Pulimento continuado de la descripción física, en particular características estereotípicas (verruga en la nariz).

- Inicio de relación entre contexto y otros elementos narrativos (contextos futurísticos para introducir alienígenas y nave espacial).

- Episodio único y lineal con un claro comienzo, medio y fin; el episodio contiene un problema, respuesta emocional, acción y resultado.

\section{Respuesta suficiente}

- Cuento organizado y claro, con cierto desarrollo.

- Generalmente, es organizado, pero tiene pocas o ninguna transición entre las partes.

- La estructura oracional puede ser simple y poco variada; generalmente, la elección léxica es la apropiada; palabras seleccionadas más cuidadosamente para ajustarse a los propósitos de la narrativa.
Como todas las evaluaciones, la del texto narrativo es subjetiva, pero sin duda la utilización de una rúbrica con descriptores detallados puede darle un carácter más objetivo.

- Los errores en gramática, ortografía y puntuación no interfieren con la comprensión.

- Motivaciones e intenciones que impulsan los sentimientos y acciones de los personajes principales a menudo a través de un punto de vista omnisciente limitado.

- El contexto se vuelve más esencial al desarrollo del'cuento de modo explícito: los personajes pueden hacer notar el contexto o el tiempo y lugar pueden ser parte esencial de la trama.

- La trama aumenta en complejidad con más de un episodio; cada episodio contiene un problema, respuesta emocional, acción y resultado.

- Información ampliada y explicaciones para el lector (vinculando ideas como también episodios).

\section{Respuesta idónea}

- Apoya el cuento con razones pertinentes y/o ejemplos en la mayor parte del texto.

- Está bien organizado, pero le faltan transiciones.

- Muestra algo de variedad en la estructura oracional y elige bien el léxico; ocasionalmente, las palabras pueden usarse en forma errónea.

- Los errores en gramática, ortografía y puntuación no interfieren con la comprensión.

- La trama cambia abruptamente en ocasiones.

- Uso de temas secundarios, a menudo vinculados a la trama mayor, pero a veces tangenciales.

- Más pulimento en sentimientos y motivaciones; aparecen características dinámicas en los personajes centrales y entre personajes.

- El contexto puede servir más de una función y la relación entre funciones es más implícita y simbólica.

- Relaciones más fuertes entre episodios, con resolución en uno de ellos que conduce a un problema en el siguiente. 
- Algo de experimentación con simbolismo (en particular, el lenguaje figurativo) que demuestra consideraciones hacia el lector.

\section{Respuesta excelente}

- El cuento se apoya en forma sistemática con razones y/o ejemplos bien elegidos; puede que use estrategias persuasivas para transmitir argumentos.

- Está focalizado y bien organizado, con un uso efectivo de transiciones.

- En forma consistente, demuestra una variedad en la estructura oracional y precisión en la elección léxica; las palabras se emplean de modo único e interesante; el autor parece reflexivo e imaginativo, aunque en partes el lenguaje utilizado sea inapropiado.

- Los errores en gramática, ortografía y puntuación son pocos y no interfieren con la comprensión.

- El autor mantiene su papel de participante y observador a través de todo el texto.

- La figura central se describe en detalle.

- La acción transcurre en un lugar bien detallado.

- El orden de eventos siempre es claro, incluso en el pasado o en el futuro.

- La trama principal es múltiple y compleja; los temas secundarios se relacionan de forma integral a los temas primarios.
- Personajes principales pulidos y dinámicos a través de la descripción rica en afecto, intención y motivación.

- El contexto se integra completamente con los personajes, acción y trama.

- Problema principal y resolución apoyados por episodios múltiples.

- Cuidadosa elección de estructura narrativa; el vocabulario también demuestra articulación razonable de todos los recursos.

Dependiendo de los resúltados que se obtengan después de la aplicación del inștrumento, los participantes serán clasificados según la taxonomía de Spalding y Cummins (1998). Esta taxonomía permite diferenciar a los participantes entre novicios, aprendices, competentes y distinguidos, y su función es, por una parte, tener una visión panorámica de los tipos de productores de textos narrativos a los que se les administró el instrumento y, por otra -más pedagógica-, obtener retroalimentación acerca de esta producción y modificar la enseñanza de este tipo de textos en caso de ser necesario.

\section{Novicio}

- Conciencia de audiencia y propósitos limitada.

- Idea de desarrollo mínima; detalles limitados y/o no relacionados.

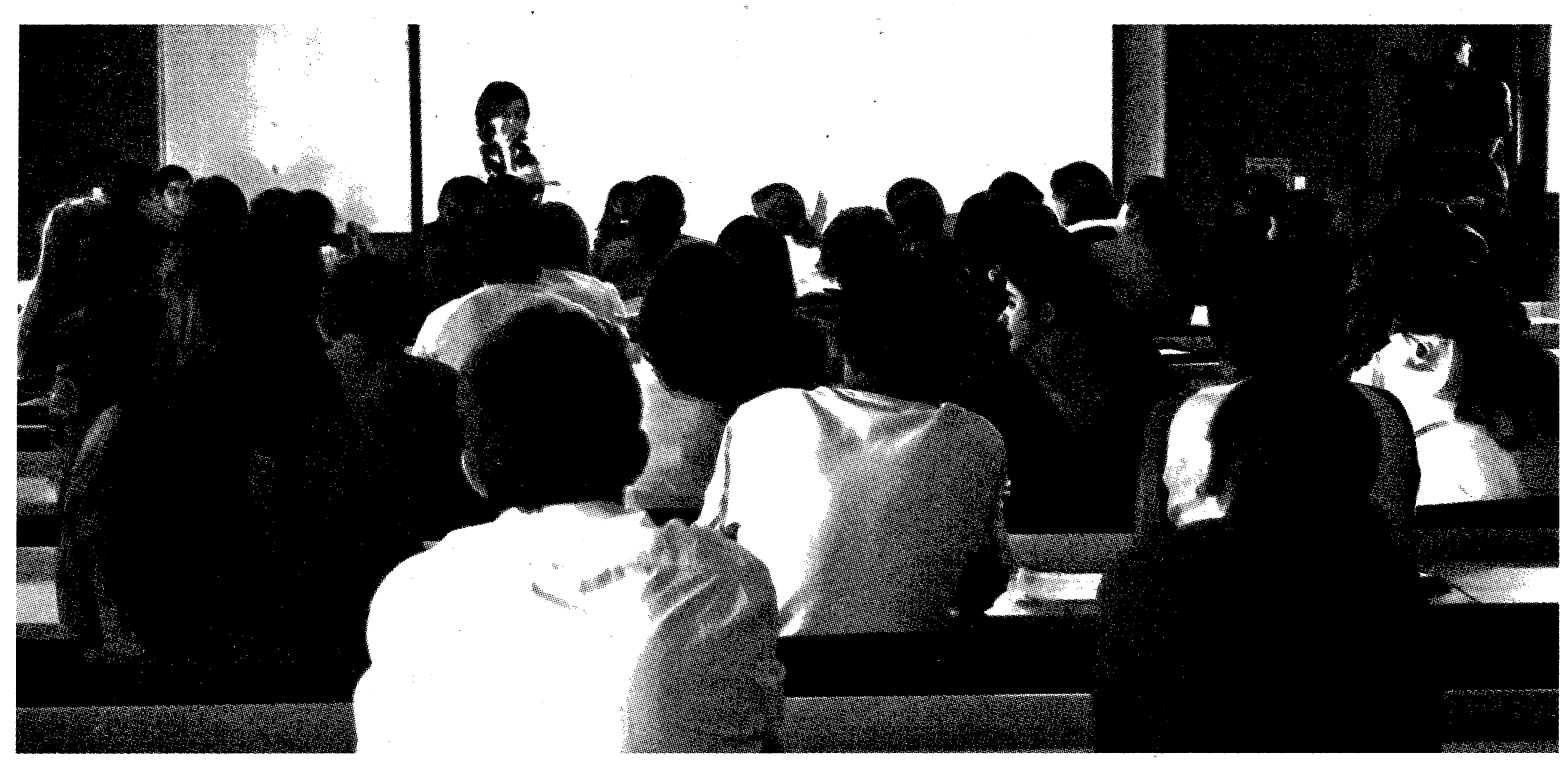


- Organización débil o al azar.

- Estructura incorrecta y/o poco efectiva.

- Lenguaje incorrecto y/o poco efectivo.

- Errores de ortografía, puntuación y uso de mayúsculas son desproporcionados a la longitud y complejidad.

\section{Aprendiz}

- Alguna evidencia de comunicación con una audiencia para un propósito específico; algunos lapsos en el foco.

- Idea de desarrollo poco elaborada; detalles poco elaborados y/o repetitivos.

- Lapsos en organización y/o cohesión.

- Estructura oracional simple y/o torpe.

- Lenguaje simple y/o impreciso.

- Algunos errores en ortografía, puntuación y uso de mayúsculas que no interfieren con la comunicación.

\section{Competente}

- Focalizado en un propósito; se comunica con una audiencia; evidencia de voz y/o tono apropiado.

- Profundidad de desarrollo de ideas apoyada con detalles elaborados y relevantes.

- Organización lógica y coherente.

- Estructura controlada y variada.

- Lenguaje aceptable y efectivo.

- Pocos errores de ortografía, puntuación y uso de mayúsculas en relación a la longitud y complejidad.

\section{Distinguido}

- Establece un propósito y mantiene un foco claro; fuerte conciencia de audiencia; evidencia de voz distintiva y/o tono apropiado.

- Profundidad y complejidad de ideas apoyada por detalles sólidos, interesantes y/o pertinentes; evidencia de análisis, reflexión y profundidad de pensamiento.

- Organización cuidadosa y/o sutil.

- Variedad de estructura y longitud oracional intensifica el efecto.

- Lenguaje preciso y/o rico.
- Control de la ortografía, puntuación y uso de mayúsculas.

\section{Conclusiones}

Como todas las evaluaciones, la del texto narrativo es subjetiva, pero sin duda la utilización de una rúbrica con descriptores detallados puede darle un carácter más objetivo. En particular, el empleo de una evaluación holística focalizada permite que el texto sea juzgado de manera general en sus cualidades retóricas (tópico sostenido, conciencia de audiencia y propósito claro) y, al mismo tiempo, que la atención del evaluador se centre en los rasgos textuales más sobresalientes que conforman una narración, como son los personajes, la acción, el contexto y el lenguaje utilizado. De igual manera, el instrumento aquí presentado desarrolla criterios para una escritura exitosa en un tipo de texto específico.

Al usarse como parte del ciclo instruccional y cuando los estudiantes escritores están concientes de los objetivos de la rúbrica y de sus descriptores, ésta los estimula a que limiten su atención a un conjunto manejable de características lingüísticas a medida que componen y revisan. En otras palabras, se puede integrar en forma natural a la enseñanza, a la composición de borradores, a la retroalimentación y al ciclo de revisiones, para producir una evaluación que informe sobre la eficiencia de acuerdo a cómo se refleje en la competencia de los estudiantes y en el logro de objetivos programáticos.

Entre los resultados esperados, podemos vislumbrar que no todas las formas y funciones de la narrativa ocurrirán, pero podemos aventurar que se compondrán narrativas noéticas y pragmáticas $y$, en un menor grado, narrativas éticas. Los estudiantes de tercero básico ya saben distinguir entre el bien y el mal, y son capaces de crear personajes que los encarnen. Sus narrativas también les permiten traducir sus experiencias en conocimiento y les ayudan a conseguir metas o fines deseados, en este caso particular, el deseo de expresarse.

La EHF ofrece una mayor validez que los métodos holísticos y analíticos propiamente, en el sentido de que tanto estudiantes como profeso- 
res perciben que mide lo que intenta medir y porque los criterios están vinculados directa y explícitamente a los objetivos de una tarea de escritura específicos. Lo anterior es una de las responsabilidades más importantes de los profesores al diseñar instrumentos de evaluación, puesto que, de lo contrario, los estudiantes se desmotivan al verse desorientados por la incongruencia entre estos últimos y los criterios empleados para evaluar.

Por último, la clasificación de los productores de textos narrativos en novicios, aprendices, competentes y distinguidos permite una diferenciación de estos productores con fines prácticos, en el sentido de que la información así obtenida puede facilitar la modificación de estrategias didácticas $y$, en último término, de programas de estudios. Ó

\section{Bibliografía}

Botting, N. (2002, febrero), "Narrative as a Tool for the Assessment of Linguistic and Pragmatic Impairments", en Child Language Teaching and Therapy, vol. 18, núm. 1, pp. 1-21.

Calderón Arévalo, M. (s.f.), "Evaluación ecológica. Evaluativa y formativa" [en línea], disponible en: http://www.rmm.cl/index_sub. php?id_seccion $=3513 \&$ id_portal $=531 \& i d$ _ contenido $=10145$

Cooper, J. D. (1990), Cómo mejorar la comprensión lectora, Madrid, Visor.

Cooper, C. y Odell, L. (1977), Evaluating Writing. Describing, Measuring, Judging, New York, National Council of Teachers of English.

Countryman, J. (1995), "Academic Narratives: What's the Story?", en Journal of the Asso- ciation for Communication Administration, núm. 1, pp. 18-27.

Denning, S. (2005), The leader's guide to storytelling, San Francisco, Wiley \& Sons, Inc.

Ferris, D. y Hedgcock, J. (1998), Teaching ESL composition: Purpose, Process, and Practice, Mahwah, NJ, Lawrence Erlbaum Associates.

George, D. (2002), "From Analysis to Design: Visual Communication in the Teaching of Writing", en College Composition and Communication, vol. 54, núm. 1, pp. 11-39.

Hamp-Lyons, L. (1991), Scoring Procedures for esL Contexts, en Hamp-Lyons L. (edit.), $A s^{-}$ sessing Second Language Writing in Academic Contexts, Norwood, NJ, Ablex, pp. 241-276.

Historias de grandes pequeños (2006), Santiago de Chile, Ministerio de Educación de la República de Chile y Grupo Océano, Fe\&Ser Ltda., pp. 20-364.

Scharton, M. (1990), Narration: A Short History, Illinois, Murphy Press.

Spalding, E. y Cummins, G. (1998), It Was the Best of Times. It Was a Waste of Time, en $A$ ssessing Writing, vol. 5, núm. 2, pp. 167-199.

White, S. y Venneman, A. (2000), “NAEP, Scoring of Fourth-Grade Narrative Writing", en Education Statistics Quarterly, vol. 2, núm. 4, pp. 1-7.

Williams, J. D. (1998), Preparing to Teach Writing: Research, Theory, and Practice, Mahwah, NJ, Lawrence Erlbaum Associates.

Wolf, S. A. y Gearhart, M. (1997, otoño), "New Writing Assessments: The Challenge of Changing Teachers' Beliefs About Students as Writers", en Theory into Practice, vol. 36, núm. 4, pp. 220-230. 